\title{
AFRONTANDO LA ENSEÑANZA DE LA EDUCACIÓN FÍSICA EN EDUCACIÓN INFANTIL: RELATO AUTOBIOGRÁFICO DE UN MAESTRO NOVEL
}

Facing the teaching of Physical Education in pre-primary stages: Autobiographical stories of a novice teacher

Enfrentando o ensino da Educação Física na educação das crianças: Conto autobiográfico de um professor iniciante

\section{Gustavo González-Calvo}

Facultad de Educación de Palencia. Universidad de Valladolid, España. Telf.: +34979108219. Correo electrónico: gustavo.gonzalez@uva.es

\section{David Hortigüela-Alcalá}

Facultad de Educación de Burgos. Universidad de Burgos, España. Telf.: +34947259517. Correo electrónico: dhortiguela@ubu.es

\section{Raúl A. Barba-Martín}

Facultad de Educación de Segovia. Universidad de Valladolid, España. Telf.: +34921112200. Correo electrónico: raulalberto.barba@uva.es

\section{Resumen}

La formación inicial debe tratar de acercar a los futuros docentes el conocimiento de los contextos reales educativos. Aun así, el maestro se puede encontrar con realidades para las que no ha sido formado. Ante esta situación, la reflexión crítica se erige como fundamental para poder subsanar las dudas y problemáticas que pueden surgir a un maestro novel. En este artículo se presenta, con una metodología autobiográfica, el modo en que un maestro novel de Educación Física se enfrenta a las consecuencias derivadas de tener que desempeñar su profesión en la etapa de Educación Infantil, una etapa para la que no se siente preparado ni cree haber tenido la suficiente formación específica como maestro de Educación Física. A través de la reflexión crítica y el diario de clase, el docente va reconstruyendo su identidad profesional al comenzar a analizar, reflexionar y poner en duda muchas de las cosas que hace y el modo en que las hace.

Palabras clave: Diarios de clase; Educación Física; Educación Infantil; formación del profesorado; relatos autobiográficos. 


\begin{abstract}
The initial teacher training should try to bring the future teachers to know the real educational contexts. Even so, the teacher may finds realities for which he has not been trained. Faced with this situation, critical reflection is fundamental to overcome the doubts and problems that may emerge to a novice teacher. This article presents, with autobiographical methodology, how a PE novice teacher faces the challenge of having to teach in pre-primary stages, something for which he has not been specifically prepared. Through critical reflection and class diary, we reflects how the teacher builds his professional identity when he start to analyze, reflect and question many of the things he does and the way in which he carries them out.
\end{abstract}

Keywords: Class diary; Physical Education; Pre-primary Education; teacher education; autobiographical stories.

\title{
Resumo
}

A formação inicial deve tentar aproximar os futuros professores do conhecimento dos contextos educativos reais. Mesmo assim, o mestre pode encontrar realidades para as quais não foi treinado. Diante desta situação, a reflexão crítica torna-se fundamental para poder corrigir as dúvidas e problemas que possam surgir em um novo professor. Este artigo apresenta, com uma metodologia autobiográfica, a forma como um novo professor de Educação Física enfrenta as consequências derivadas de ter de exercer a sua profissão na etapa de Educação Infantil, etapa para a qual não se sente preparado nem acredita ter tido formação específica suficiente como professor de Educação Física. Através da reflexão crítica e do diário de aula, o professor reconstrói a sua identidade profissional, começando por analisar, reflectir e questionar muitas das coisas que faz e a forma como as faz.

Palavras-chave: Diário do professor; Educação Física; Educação Infantil; Formação de Professores; Histórias Autobiográficas.

\section{Introducción}

Uno de los aspectos fundamentales que define la calidad de la docencia y la identificación del docente con los modelos pedagógicos implícitos en su práctica profesional es la formación inicial recibida (Hagenauer \& Volet, 2014). Por ello, es importante que los contenidos de las titulaciones cubran las necesidades de un futuro 
docente, quien ha de adquirir conocimiento relacionado con el desempeño profesional y los contextos educativos reales (Hortigüela, Salicetti, \& Pérez-Pueyo, 2015).

Es evidente que los primeros años de un maestro novel son de adaptación, aprendizaje y resolución de problemas profesionales, éticos y personales (Cantón, Cañón, \& Arias, 2013). Sin embargo, la estructura de la formación inicial del futuro maestro ha de enfocarse con el fin de producir el menor impacto y disonancia entre las expectativas del profesional y la realidad educativa, siendo para ello favorable el uso de metodologías reflexivas y el fomento de la conciencia crítica (Karabulut, 2012).

Desde el ámbito pedagógico y educativo se ha de ser consciente de la influencia que tiene el desarrollo psicoevolutivo del alumnado, sus características personales y los entornos familiares para secuenciar propuestas de aprendizaje que sean verdaderamente significativas (Sarmiento, Andrés, \& Van Gameren, 2015). Es por ello que la formación ha de ser específica para cada etapa en la que se imparte docencia, desarrollando propuestas curriculares que verdaderamente aporten y contribuyan a la adquisición de contenidos, valores y emociones (Kimura, 2010). Esto contrasta con la realidad actual, donde en muchos casos el maestro se convierte en un ser camaleónico que ha de impartir clase en diferentes materias, secciones e incluso etapas, lo que provoca, en algunos casos, un sentimiento de indefensión y saturación (Goodwin \& Webb, 2014).

\section{Los procesos reflexivos críticos. Una herramienta de formación docente}

La mejor herramienta para complementar el conocimiento adquirido en la formación inicial del docente, además de la formación permanente, es la reflexión personal sobre los acontecimientos surgidos en el día a día, utilizando para ello instrumentos de corte cualitativo que permitan buscar pautas de mejora a través de ciclos de investigaciónacción (Barrett, 2007). Es imprescindible que el docente tenga la capacidad de posicionarse bajo un enfoque crítico, abierto y comprensivo que haga entender las problemáticas encontradas como un medio para la mejora y el aprendizaje, algo que incrementará su capacidad de resiliencia y tolerancia a la frustración. Como indican Parsons y Vaughn (2013) la realidad contextual es la que provoca que el docente adapte específicamente aquello que quiere aportar a la comunidad educativa.

Si partimos de la base de que la educación ha de constituirse bajo parámetros de actuación abiertos, democráticos y mejorables a partir de las experiencias y resultados de aprendizaje, es prioritario que el docente sea capaz de reflexionar sobre su práctica y 
actuar en consecuencia, ya que es la única manera de poder avanzar, innovar, solucionar problemas y como consecuencia, mejorar la calidad de la educación (Heineke \& Preach, 2013). Por lo tanto, es necesario que desde la formación inicial del profesorado se trabajen estos aspectos, demostrándose que son decisivos en la satisfacción del desempeño profesional. Para ello, es preciso recordar que no nos encontramos solos antes estos interrogantes, ya que los compañeros, el propio centro y la comunidad educativa han de trabajar en una misma dirección, creando climas de aprendizaje constructivos y emocionalmente positivos.

\section{La enseñanza de la Educación Física en Educación Infantil}

La enseñanza de la EF requiere de mucha experiencia y conocimientos prácticos por parte del docente sobre los contenidos de la materia para poder llevar a cabo una enseñanza eficaz de los mismos (Dodds, 1994). Si el docente tiene un conocimiento limitado acerca de estos contenidos, como puede ser el caso del profesor novel, el proceso de enseñanza/aprendizaje se ve afectado y resulta poco educativo (Tan, 1996).

En este sentido el concepto de enseñanza, tal y como lo entiende Passmore (1983), supone una relación triádica entre el sujeto que enseña, alguien a quien enseña y una materia a enseñar. La enseñanza ha de ir encaminada hacia la búsqueda de unos aprendizajes sólidos y transferibles.

El concepto anterior de enseñanza sirve para evidenciar una de las preocupaciones recurrentes del docente: intentar enseñar a los estudiantes contenidos en los que su dominio es menor, donde sobreviene el temor de fracasar en la tarea de enseñar. Esta situación sitúa al docente novel en un estado de choque o shock con la práctica real (Eirín Nemiña, García Ruso, \& Montero Mesa, 2009; Esteve Zarazaga, 1993, 1997; Flores, 2009; Marcelo García, 2009a, 2009b; Teixidó Saballs, 2009; Veenman, 1988; Vezub, 2007) que genera algunos problemas a la hora de saber dar respuesta al ajuste entre el contenido a enseñar, el alumno y la labor docente (González Calvo \& Fernández Balboa, 2016).

Esta problemática se ve acrecentada en el trabajo del movimiento y lo corporal en el ámbito de infantil, en el que el desarrollo de la psicomotricidad carece de regulación profesional en cuanto a la formación específica del docente que la imparte. En la mayoría de los casos lo hace cualquier docente de la etapa, preferiblemente si es especialista en el área de Educación Física (EF) en Primaria. Sin duda, este hecho 
parece una incoherencia pedagógica, ya que la idiosincrasia de la psicomotricidad en la primera fase educativa del niño (indagación, descubrimiento, interacción lúdica,...) difiere en gran parte de los objetivos que regulan la EF en la etapa de primaria. Esto conlleva una readaptación de los esquemas cognitivos del docente, que ha de buscar fórmulas de actuación que no tienen conexión directa con la formación académica recibida (Patton \& Griffin, 2008).

El principal objetivo de este estudio es profundizar en el modo en el que un docente novel, formado en Educación Física en Primaria, ha de enfrentarse al desempeño de su profesión en Educación Infantil, una etapa en la que no se siente preparado. Para ello se refleja el sentir de sus emociones y el modo en el que va cambiando su forma de abordar la situación.

El docente, descubre en la reflexión crítica una forma de poder avanzar en su formación y subsanar la problemática de ser un maestro novel en una etapa para la que no se formó. Todo ello se presenta mediante un enfoque autobiográfico, lo que permite adentrarse y comprender mejor la intrahistoria del contenido. Esto supone un aporte significativo a la literatura existente en la temática del estudio.

\section{Metodología}

\section{Participantes}

El protagonista de nuestra investigación es un docente de EF que ha trabajado en Educación Infantil, Educación Primaria, Educación Secundaria y en la Universidad. En los últimos tres años su labor se ha centrado principalmente en la etapa de Educación Primaria.

Aunque se encontraba cursando la carrera universitaria en Ciencias Químicas, por diferentes motivos (ver González Calvo, 2013; González Calvo \& Martínez Álvarez, 2009) cambió esa orientación y comenzó sus estudios de maestro de EF en el año 2003, cuando ya contaba con 25 años.

La influencia de algunos profesores de formación inicial fue determinante a la hora de iniciarse en la producción de relatos de corte autobiográfico como método de análisis de su práctica docente, en los que actúa como investigador e investigado de manera simultánea. Con los años, este método de análisis autobiográfico se ha ido convirtiendo en un hábito, dando lugar a diferentes investigaciones y a la presentación 
de su tesis doctoral (González-Calvo \& Fernández-Balboa, 2016; González Calvo, 2013; González Calvo \& Barba, 2013; González Calvo \& Becerril González, 2013), en la que analiza el modo en que va evolucionando su identidad profesional.

La elección de nuestro educador se justifica en tanto que estamos convencidos de que representa un modelo relevante de lo que supone la formación inicial en el proceso de reconstrucción de la identidad profesional docente.

\section{Instrumentos}

El instrumento más utilizado para la producción y extracción de los relatos autobiográficos es el diario de clases. Zabalza (2008, p. 15) afirma que los diarios "son los documentos en los que profesores y profesoras recogen sus impresiones sobre lo que está sucediendo en sus clases" (p. 15). Además, son un instrumento de registro de experiencias educativas con la intención de ser posteriormente analizadas y compartidas (Smyth, 1991).

Distintos autores (Barba, González Calvo, \& Barba Martín, 2014; Chacón Corzo \& Chacón Contreras, 2006; González Calvo \& Barba, 2014; González Calvo \& Becerril González, 2013; Liston \& Zeichner, 2003; Surbeck, Han, \& Moyer, 1991; Zabalza Beraza, 2008; Zeichner \& Liston, 1999) resaltan que los diarios constituyen una herramienta motivadora de procesos críticos y reflexivos, que ayudan a ampliar el pensamiento acerca de los acontecimientos que tienen lugar en el aula y posibilitan que los docentes profundicen en sus propias acciones e ideas sobre los procesos de enseñanza/aprendizaje.

\section{Proceso investigador}

El empleo del diario de clases como instrumento de recogida de información es previo a la realización de la tesis doctoral de la que forma parte el estudio que aquí presentamos. La primera experiencia con esta herramienta aparece en la etapa de formación inicial como maestro especialista de EF, en el año 2005. Esta primera incursión fue suficiente para que el docente comprobara su utilidad para profundizar en su docencia, detectar los puntos débiles en su actuación y, así, reorientar la enseñanza a partir de la observación y el análisis llevado a cabo. En este sentido, el diario es un instrumento de formación, pero también de investigación, al permitir la recogida de datos significativos, la reflexión sobre los mismos, su análisis y sistematización. Es por estas razones, que el 
educador continúa empleando sistemáticamente este instrumento de recogida de información.

El criterio general que sigue el educador es dejar constancia de todo aquello que le resulta interesante para mejorar en su profesión y en su vida en la escuela. A este particular, tanto la identidad profesional como las reflexiones en el diario han ido evolucionando paulatinamente: de unos inicios fuertemente marcados por atender a lo curricular y lo estructural de la clase, va erigiéndose en un docente más preocupado por atender a lo personal de los alumnos y a sus preocupaciones como educador, tomando el diario un carácter más autoexpresivo. Se va convirtiendo así en una constante referencia a los alumnos, a lo que cada uno de ellos hace y el modo en que va evolucionando, y donde también tienen cabida las referencias al propio profesor, a cómo se siente y cómo actúa.

Destacar el carácter diario e individual de estos escritos, donde se destacan los aspectos más relevantes acontecidos en el aula y en el centro escolar. Queremos puntualizar que, el hecho de que la recogida de datos sea individual, no implica que el análisis de los mismos lo sea. Se ha garantizado una triangulación de investigadores: los datos han sido interpretados y enriquecidos con la puesta en común de varios investigadores expertos en el ámbito de la Didáctica de la EF y la Pedagogía, incrementando la calidad y la validez de los mismos al excluir el sesgo de un único investigador (Devís \& Sparkes, 2004; Sparkes, 1993).

Debemos señalar que el análisis de la información se ha llevado paralelamente al proceso investigador. A lo largo del tiempo se han ido recogiendo diferentes datos, escritos, opiniones, narraciones, que han facilitado profundizar en lo vivido $\mathrm{y}$ acontecido por el docente. Ésta es una práctica habitual en investigación cualitativa (Devís \& Sparkes, 2004), en la que "la recolección y el análisis de los datos van de la mano" (Taylor \& Bogdan, 1998, p. 158).

\section{Un extraño en un mundo desconocido: Aprendiendo a ser maestro de Educación}

\section{Física en Educación Infantil}

En la educación en general, y en la etapa de Educación Infantil en particular, se ha de proporcionar una serie de experiencias a los escolares que estimulen su desarrollo personal completo. Por ello, se ha de ofrecer espacios de libertad y movilidad al alumnado que permitan no solo ofrecer posibilidades de expresión propias de la 
infancia, sino que da información al docente sobre quién es el niño y permite, de esta forma, conocerlo mejor desde quién él es (Martínez Álvarez \& González Calvo, 2016). En Educación Infantil en particular, dar cabida al ámbito de lo corporal es tanto más importante en cuanto que posibilita que los alumnos conozcan su cuerpo y el de los demás $\mathrm{y}$, desde los primeros años de vida, desarrollen actitudes no discriminatorias y socializadoras.

Nuestro educador, dentro de los estudios conducentes a la titulación de maestro especialista en Educación Física, tuvo la oportunidad de formarse en la pedagogía corporal dentro de la etapa de Educación Infantil. Esta formación inicial le preparó para desempeñar su labor con el alumnado de tres a cinco años, estableciendo un puente entre teoría pedagógica y práctica en el aula que permitió suavizar las situaciones problemáticas respecto a los contenidos que tendría que impartir y el shock con la realidad tan habitual entre educadores inexpertos (González-Calvo \& FernándezBalboa, 2016; González Calvo, Barbero González, Bores Calle, \& Martínez Álvarez, 2014). No obstante, y puesto que la titulación de Educación Física va enfocada, fundamentalmente, a la etapa de Educación Primaria y no tanto a la de Educación Infantil, se aprecia en el docente una mayor inseguridad y un mayor desconocimiento de lo que ha de enseñar al alumnado de tres a cinco años respecto al alumnado de seis a doce años, con quienes demuestra una mayor competencia:

"Las clases con los pequeños son eternas. Se me terminan los recursos que llevaba preparados, los niños se cansan pronto de las tareas propuestas y entonces empieza a crecer en mí la idea de que, otra vez, voy a tener que rellenar parte de la lección con el "hacer por hacer". Me siento incapaz de enseñar nada, creo que lo que hago bien lo podría hacer cualquiera y, entonces, ¿de qué sirve que yo haya estudiado?, ¿y que me esté leyendo libros sobre Psicomotricidad?" (Diario del profesor. Octubre de 2009).

Al mismo tiempo, el educador compara su práctica con la literatura científica, llegando a pensar que las propuestas que puede hacer en esta etapa son, cuando menos, utópicas:

"Los libros que utilizo como referentes parecen ideales, es el "mundo de Oz” y para mí, sin embargo, es todo lo contrario. A veces espero leer alguna frase del tipo: "A mis inicios, era un completo desastre y no sabia ni 
lo que hacía”, pero esa frase nunca llega. Todo el mundo sabe y, el que no, al menos se reserva el lujo de opinar cómo debería enfocar las clases. Pero yo no sé, no he recibido una formación específica en torno a la Educación Infantil, y si estoy dando clase en este nivel es porque de alguna manera había que rellenar mi horario." (Diario del profesor. Octubre de 2009).

Relativizar y restar importancia al hecho de no saber cómo enfocar las clases y cómo lograr aprendizajes con los más pequeños lleva a atravesar por momentos en los que se pasa por la cabeza renunciar y rendirse ante la evidencia de que, con la formación recibida, el docente no está preparado en absoluto para cumplir con sus responsabilidades:

"Con los de infantil es frecuente que se me pase por la cabeza pensar en tirar la toalla ("total, si voy a estar en este cole un año y únicamente tengo con ellos una clase a la semana, ¿para qué me voy a llevar malos ratos”). Además, como maestro en una escuela rural, debo añadir otro inconveniente: al ser un colegio pequeño, el agrupamiento de alumnos se hace por ciclos. En el caso de Infantil, tengo alumnos de 3, 4 y 5 años, y aunque a priori podría parecer poca diferencia de edad en realidad supone tener unos alumnos muy heterogéneos. No sé si sería capaz de mejorar mi práctica con alumnos de la misma edad, pero al menos sí creo que me resultaría más sencillo intentarlo. Uno sólo puede hacer aquello de lo que es capaz. En mi caso, sobrevivir a la sesión de Psicomotricidad como buenamente pueda. Menos mal que hasta dentro de una semana no tengo otra" (Diario del profesor. Noviembre de 2009).

Se presenta una evidente dificultad cuando el educador ha de presentar los aprendizajes a estudiantes de niveles diferentes y, por lo tanto, con necesidades y capacidades distintas. La heterogeneidad de los escolares complica más la situación para un maestro novel cuya formación no ha sido específica de la educación en estas edades, de lo que deriva el desconocimiento de qué tipo de contenidos y aprendizajes son los apropiados para este alumnado y cómo éstos se pueden abordar de forma productiva.

No obstante, y a pesar de los múltiples inconvenientes, el maestro comienza a reflexionar y a entender que algunos de esos obstáculos pueden reconducirse y entenderse como ventajas. Surge así la idea de aprovechar que los estudiantes de mayor 
edad puedan establecer unas relaciones provechosas con los más pequeños. De este modo, y bien avanzado el curso, el maestro opta por ayudarse de estos alumnos más mayores e instruirlos, en cierto modo, en la enseñanza de los más pequeños:

"Llevo ya algunas semanas observando, fundamentalmente en el horario del recreo, que los pequeños imitan las actuaciones de los mayores. Estos últimos, sin embargo, no suelen ser conscientes de ello, sus respuestas egocéntricas y su corta edad no favorecen unas relaciones sociales fructiferas entre ellos. Se me ocurre pensar si yo podría intentar ayudarme de esta situación, si no serían más capaces estos alumnos, por ser el modelo en que se reflejan los niños de tres y cuatro años, quienes mejor podrían enseñarles. Seguro que es una tarea complicada, pues requiere que los niños de cinco años sean conscientes de sus respuestas, de sus pensamientos para, así, poder explicárselo a su vez a los menores. Pero intentaré ponerlo en práctica en la siguiente clase" (Diario del profesor. Marzo de 2010).

Es en estos procesos en los que el aprendizaje tiene una transferencia más evidente a las situaciones cotidianas de los estudiantes (Barba Martín, 2008), ya que no basta con aprender un determinado contenido, sino que se aprende a analizar, pensar y evaluar ese contenido. Al mismo tiempo, este tipo de trabajo mejora las relaciones personales entre el grupo:

"Hemos comenzado realizando algún juego propuesto y explicado por los alumnos más mayores. En gran grupo, se ha establecido un diálogo en el que los que más saben explicaban y ayudaban a los que más dificultades tenían. Por otra parte, los juegos propuestos debían ser variados, recogiendo los intereses de todos. Creo que trabajar de esta manera favorece que los niños se sientan no sólo más responsables y dispuestos hacia el aprendizaje, sino también más queridos y escuchados dentro de la clase" (Diario del profesor. Marzo de 2010).

Procurar dotar al alumnado de Educación Infantil de cierta autonomía puede ser visto como una práctica utópica. Los adultos tendemos a pensar que los niños no son capaces de implicarse en lo que hacen, que debido a su corta edad no disponen de una concentración que les permita llevar a cabo una tarea como la de explicar un juego a sus iguales. La práctica demuestra lo contrario a nuestro educador: 
"La semana pasada pedí a dos de las alumnas mayores que trajeran pensados diferentes juegos para poder explicárnoslos a los demás. Después de la conversación con algunas de mis compañeras pensaba que no se acordarían. Sin embargo, no sólo sí se acordaron, sino que trajeron escrito en una ficha el desarrollo de la actividad. Cuántas veces tendemos a infravalorar el potencial de los niños. Es fundamental, en el ámbito de la educación, que los maestros aprendamos a respetar a los escolares por lo que son y por cómo son, valorando sus acciones, sus pensamientos, sus reflexiones, sus inquietudes que, si bien es cierto que son menos complejas que las de los adultos, no son inexistentes". (Diario del profesor. Abril de 2010).

Esta situación lleva a que el docente se sienta mucho más seguro y confiado con su práctica pedagógica. Si bien no termina de estar seguro de proporcionar aprendizajes a los escolares, sí cree estar educando en valores y consiguiendo un acercamiento más afectivo con el alumnado:

"Me siento mucho más satisfecho en las sesiones desarrolladas en las últimas semanas. No sé si estoy consiguiendo un aprendizaje entre el alumnado; pero sí considero que los niños se están sintiendo más escuchados, más valorados dentro del aula. Se les debe ofrecer la oportunidad de sentirse importantes y parte del grupo. Quizá sea éste el punto de partida del docente, el punto de partida para lograr un proceso de enseñanzalaprendizaje provechoso y positivo" (Diario del profesor. Abril de 2010).

Tanto es así que el educador se muestra convencido de que, si su futuro profesional le vuelve a acercar al mundo de la Educación Infantil, tratará de partir de la creación de un clima de aula en el que todo el alumnado tenga la oportunidad de hacer y decir, de probar y sentirse escuchado:

"Puede que los inicios tan complicados con el alumnado de Educación Infantil se hayan debido, en parte, a que mis propuestas eran demasiado cerradas, donde únicamente esperaba que ellos hicieran lo que yo les mandaba. Es cierto que yo también he infravalorado la capacidad de los pequeños, e incluso es posible que haya confundido, en diferentes momentos 
del curso, la enseñanza con el adiestramiento. Si mi idea de educación está fundamentada en el desarrollo de unos valores en los estudiantes, entre otros objetivos, ¿por qué no ha sido así desde el comienzo con estos escolares? Por otra parte, si con los alumnos de edades más avanzadas he utilizado unos estilos de enseñanza menos directivos, ¿por qué con éstos no he dejado oportunidades para que se equivoquen, exploren, aprendan, vayan conociendo sus posibilidades motrices y también sus limitaciones? Supongo que gran parte de la culpa la haya tenido el miedo a que estos alumnos pudieran hacerse daño dentro de mis sesiones. Al fin y al cabo, yo soy el responsable de su integridad fisica" (Diario del profesor. Junio de 2010).

En la actualidad, esa realidad educativa ha vuelto a acercar al docente a las aulas de Educación Infantil. En este sentido, el educador sigue considerando que el tratamiento que ha recibido durante su formación inicial respecto a la materia de EF en la etapa de Educación Infantil ha sido escaso, tanto en tiempo como en contenidos. De esta manera entiende que, si bien es cierto que en principio los especialistas de EF van a desarrollar su labor, principalmente, en la etapa de Educación Primaria, quizá sería conveniente también una estructuración de los estudios iniciales que ofrezca a dichos contenidos una cierta uniformidad y seriedad.

Por otro lado, en este momento otorga una mayor importancia a la etapa de Educación Infantil. Coincide con Zabalza (1996) al considerarla una etapa en la que al niño se le ofrece una variada gama de estímulos y oportunidades para desarrollar una serie de aprendizajes cognitivos, éticos, sociales y físicos, de manera que el alumno pueda alcanzar su máximo desarrollo potencial.

Compartir estos planteamientos le lleva a sentirse, hoy día, como un docente con una mayor sensación de seguridad y de competencia en lo que hace, más confiado y cómodo en el aula.

\section{A modo de epílogo}

A la vista de los resultados presentados en nuestro estudio inferimos que la formación inicial cumple con una importante función en el avance de la identidad profesional del docente novel. No en vano, la principal referencia del educador en sus inicios en la profesión no son únicamente sus experiencias vitales previas relacionadas con la 
enseñanza, sino también la formación inicial recibida, a partir de la cuál va construyendo y desarrollando su propio conocimiento pedagógico. Asimismo, es en esta etapa en la que se desvelan la pasión y la ilusión por esta profesión, descubriendo el significado de lo que es ser profesor, pues éste llega a la formación inicial sin una identidad docente formada (recordar que accede a los estudios por el vínculo que asocia a éstos con la salud, no con aspectos educativos). Por lo tanto, consideramos que una preparación inicial conveniente y apropiada a la realidad de las aulas suaviza las situaciones problemáticas respecto a los contenidos que ha de impartir y el shock con la realidad tan habitual entre los educadores inexpertos (Eirín Nemiña et al., 2009; Esteve Zarazaga, 1993, 1997; Flores, 2009; Marcelo García, 2009a, 2009b; Teixidó Saballs, 2009; Veenman, 1988; Vezub, 2007).

Pese a ello, apreciamos una situación problemática en el momento en que el educador ha de desempeñar tareas para las que no ha recibido una adecuada formación. Tal es el caso de la enseñanza en la etapa de Educación Infantil, para la que el maestro no ha sido debidamente preparado y en la que ha de enfrentarse a muchas dificultades. Nos reafirmamos, a la luz de los resultados, en la idea de que la inexistencia de una correlación entre la formación recibida y la plaza que se ocupa en el centro escolar implica una situación problemática. En este sentido, es imprescindible que el maestro especialista en Educación Física sea formado en la etapa de Educación Infantil, pues es principalmente a través de esta materia por la que se pueden desarrollar y trabajar los valores importantes para el desarrollo personal e integral de los alumnos, ya que la Educación Física no es algo aislado, sino que está condicionada y debe servir al desarrollo corporal y motor del alumno y también a su desarrollo cognitivo y afectivo para así lograr su formación íntegra.

La escasa formación inicial recibida y la incapacidad para responder a las demandas de la profesión hacen que la identidad profesional que va desarrollando nuestro docente se vea afectada negativamente, haciendo mella en su condición como profesional de la educación e incluso llegando a adoptar estrategias de ego-protección con las que hacer frente a sus carencias. En este punto, si bien en el análisis de los datos se aprecia cierto crecimiento como maestro en la etapa de Educación Infantil, también se demanda una reestructuración de los estudios iniciales de Educación Primaria que ofrezcan al educador la posibilidad de enfrentarse a esta etapa de manera seria y competente. De lo contrario, se corre el riesgo que se aprecia en nuestro estudio: el de 
una completa sensación de falta de conocimiento de los contenidos que ha de enseñar y, por consiguiente, la convicción de que ha de procurar alejarse, en lo sucesivo, de la enseñanza en las edades de infantil.

Sin embargo, el devenir profesional le lleva a probar y dar un giro a su práctica pedagógica, de manera que sea posible ofrecer una mayor responsabilidad a los escolares dentro de las lecciones. Eso, conjuntamente con una mayor autonomía y profesionalidad fruto de su mayor experiencia dentro de las aulas, le llevan a saber gestionar mejor las relaciones interpersonales con los alumnos de la etapa de Educación Infantil.

A día de hoy, el educador sigue ejerciendo como maestro de Educación Infantil a tiempo parcial, reforzando esa idea de que es, en esta etapa, cuando ha de fomentar aprendizajes capaces de desarrollar la iniciativa, la creatividad y la motricidad de los más pequeños.

En todo caso, queremos recordar que la identidad profesional docente no es estanca, fija e inamovible, sino algo que forma parte de un todo del que el educador ha de ir aprendiendo y reconstruyendo en pro de mejorar su práctica pedagógica día a día.

\section{Referencias bibliográficas}

Barba, J. J., González Calvo, G., \& Barba Martín, R. (2014). El uso de los diarios del profesorado como instrumento de reflexión-sobre-la-acción. Revista Española de Educación Física y Deporte (REEFD), 405, 55-63.

Barba Martín, J. J. (2008). La formación del maestro novel mediante la investigaciónacción. El camino hacia la construcción de unas prácticas críticas y coherentes. In A. Gutiérrez Martín \& L. Torrego Egido (Eds.), Participatory action research as a necesary practice for the twenty-first century society (pp. 39-42). Manchester: Manchester Metropolitan University (CARN).

Chacón Corzo, M., \& Chacón Contreras, A. (2006). Los diarios de prácticas: una estrategia de reflexión en la formación docente. Acción Pedagógica, 15, 120127.

Dodds, P. (1994). Cognitive and behavioral components of expertise in teaching Physical Education. Quest, 46(2), 153-163. 
Eirín Nemiña, R., García Ruso, H. M., \& Montero Mesa, L. (2009). Profesores principiantes e iniciación profesional. Estudio exploratorio. Revista de Currículum y Formación de Profesorado, 13(1), 101-115.

Esteve Zarazaga, J. M. (1993). El choque de los principiantes con la realidad. Cuadernos de Pedagogía, 220, 58-63.

Esteve Zarazaga, J. M. (1997). La formación inicial de los profesores de Secundaria. Barcelona: Ariel.

Flores, M. A. (2009). La investigación sobre los primeros años de enseñanza: lecturas e implicaciones. In C. Marcelo García (Ed.), El profesorado principiante: inserción a la docencia (pp. 59-98). Madrid: Octaedro.

González-Calvo, G., \& Fernández-Balboa, J. M. (2016). A qualitative analysis of the factors determining the quality of relations between a novice physical education teacher and his students' families: implications for the development of professional identity. Sport, Education and Society, 1-14. doi:10.1080/13573322.2016.1208164

González Calvo, G. (2013). Evolución de la identidad profesional de un docente novel de Educación Física: análisis a partir de la reflexión de sus experiencias personales y de su propia práctica. Tesis Doctoral. Universidad de Valladolid, Valladolid.

González Calvo, G., \& Barba, J. J. (2013). La perspectiva autobiográfica de un docente novel sobre los aprendizajes de Educación Física en diferentes niveles educativos. Cultura, Ciencia y Deporte, 8(24), 171-181.

González Calvo, G., \& Barba, J. J. (2014). Formación permanente y desarrollo de la identidad reflexiva del profesorado desde las perspectivas grupal e individual. Profesorado: Revista de Currículum y Formación del Profesorado, 18(1), 398412.

González Calvo, G., Barbero González, J. I., Bores Calle, N., \& Martínez Álvarez, L. (2014). (Re)construction of a teacher's professional identity from his initial training: Autobiographical narration. The Open Sports Science Journal, 7(2), 113-120. 
González Calvo, G., \& Becerril González, R. (2013). El recorrido investigador de un educador novel explicado desde una perspectiva autobiográfica. Revista Electrónica Interuniversitaria de Formación del Profesorado, 16(3), 61-78.

González Calvo, G., \& Martínez Álvarez, L. (2009). Aproximación a los significados e interpretaciones de la lesión en futuros docentes de Educación Física por medio de narraciones autobiográficas. Retos: Nuevas Tendencias en Educación Física, Deporte y Recreación, 15(46), 35-40.

Liston, D. P., \& Zeichner, K. M. (2003). Formación del profesorado y condiciones sociales de la escolarización. Madrid: Morata.

Marcelo García, C. (2009a). El profesorado principiante: inserción a la docencia. Barcelona: Octaedro.

Marcelo García, C. (2009b). Los comienzos en la docencia: un profesorado con buenos principios. Profesorado, revista de currículum y formación del profesorado, $13(1), 1-26$.

Martínez Álvarez, L., \& González Calvo, G. (2016). Docentes de carne y hueso: enseñar con cuerpo. Ágora para la Educación Física y el Deporte, 18(3), 259275.

Smyth, J. W. (1991). Una pedagogía crítica de la práctica en el aula. Revista de Educación, 294, 275-300.

Surbeck, E., Han, E., \& Moyer, J. (1991). Assessing reflective responses in journals. Educational Leadership, 48(6), 25-27.

Tan, S. K. S. (1996). Differences between experienced and inexperienced Physical Education teachers' augmented feedback and interactive decisions. Journal of Teaching in Physical Education, 15(2), 151-170.

Teixidó Saballs, J. (2009). La acogida al profesorado de nueva incorporación. Barcelona: Graó.

Veenman, S. (1988). Perceived problems of beginning teachers. Review of Educational Research, 54(2), 143-178. 
Vezub, L. F. (2007). La formación y el desarrollo profesional docente frente a los nuevos desafíos de la escolaridad. Profesorado, revista de currículum y formación del profesorado, 11, 2-33.

Zabalza Beraza, M. A. (1996). Didáctica de la Educación Infantil. Madrid: Narcea.

Zabalza Beraza, M. A. (2008). Diarios de clase: un instrumento de investigación y desarrollo profesional. Madrid: Narcea.

Zeichner, K. M., \& Liston, D. P. (1999). Enseñar a reflexionar a los futuros docentes. In A. Pérez Gómez, J. Barquín Ruiz, \& J. F. Angulo Rasco (Eds.), Desarrollo profesional del docente. Política, investigación y práctica (pp. 506-532). Madrid: Akal. 